\title{
Usefulness of cardiac resynchronisation therapy in patients with right bundle branch block: Is viability an important piece of the puzzle?
}

\author{
K. O’Connor ${ }^{a}$, M. Sénéchal ${ }^{\text {a,* }}$, P. Lancellotti ${ }^{b}$, M. Dubois ${ }^{a}$, J. Magne ${ }^{\text {a }}$, J. Champagne ${ }^{a}$, \\ F. Philippon ${ }^{\mathrm{a}}$, L. Pierard ${ }^{\mathrm{b}}$, G. O'Hara ${ }^{\mathrm{a}}$ \\ a Institut de Cardiologie de Québec, Hôpital Laval, Department of Cardiology, Québec, Canada \\ ${ }^{\mathrm{b}} \mathrm{CHU}$ de Liège, Department of Cardiology, University Hospital, Sart Tilman, Liège, Belgium
}

Received 26 September 2008; accepted 14 December 2008

\begin{abstract}
Background: Benefits of cardiac resynchronisation therapy (CRT) in patients with heart failure (HF) and left bundle branch block (LBBB) have been well established. The presence of asynchronism and viability predicts response to CRT with good accuracy. Viability in the region of the pacing lead as predictor of response to CRT in patients with HF, intraventricular asynchrony and right bundle branch block (RBBB) has never been evaluated.

Methods: We studied 4 consecutive patients with RBBB (QRS $>120 \mathrm{~ms}$ ) advanced ischemic HF, low ejection fraction ( $\leq 35 \%)$ and intraventricular asynchrony $\geq 50 \mathrm{~ms}$ scheduled for CRT. Dobutamine stress echocardiography (DSE) was performed within the week before CRT. Viability was defined as increased wall thickening during DSE. Viability in the region of left ventricular (LV) pacing lead was defined as the presence of viability in 2 contiguous segments. Response was defined by LV reverse remodeling (i.e. $\geq 15 \%$ reduction in LV endsystolic volume) 3-6 months after CRT.

Results: Three patients demonstrated LV reverse remodeling at follow-up. Responders showed LV end-systolic volume decrease of $-31 \pm 16 \%$ from baseline to follow-up whereas no change was observed in the non responder patient. Similar LV asynchronism was found in all patients. All responders had viability in $\geq 2$ segments in the region of LV pacing.

Conclusion: This preliminary report suggests that similar reverse remodeling can be observed in RBBB patients as patients with LBBB after CRT. Intraventricular asynchrony and RBBB, viability in the region of pacing lead may help to predict response to CRT in patients with HF. (C) 2008 Elsevier Ireland Ltd. All rights reserved.
\end{abstract}

Keywords: CRT; RBBB; Asynchronism; Viability

\section{Introduction}

ACC/AHA Guidelines recommended cardiac resynchronization therapy (CRT) in patients with heart failure (HF) with New York Heart Association (NYHA) class III to IV, low ejection fraction $(\leq 35 \%)$ and $\mathrm{QRS}$ duration $>120 \mathrm{~ms}$, regardless of intraventricular delay pattern [1]. Despite these

\footnotetext{
* Corresponding author. Institut de Cardiologie de Québec, Hôpital Laval, 2725, Chemin Sainte-Foy, Québec, Québec Canada G1V 4G5. Tel.: +1 418 656 8711; fax: +1 4186564581 .

E-mail address: mario.senechal@crhl.ulaval.ca (M. Sénéchal).
}

criteria, up to $40 \%$ of patients failed to demonstrate a response to CRT [2]. Over $90 \%$ of patients enrolled in CRT studies have left bundle branch block (LBBB). In those patients, LV dyssynchrony helps to predict response to CRT [3]. Presence of viability has also gained growing acceptance as a predictor of response to CRT in patients with HF $[2,4-6]$.

Less than $10 \%$ of patients with advanced systolic HF and abnormal ventricular conduction have RBBB. Consequently, patients with RBBB represent a small proportion of patients enrolled in CRT studies and limited data are available in this particular population [7]. In 2001, Garrigue et al. showed in 12 patients that only patients with RBBB associated with 
major intraventricular asynchrony are likely to respond to CRT [8]. However recently, Egoavil et al. analyzed the combined results of MIRACLE and Contak CD trials, which provided the largest cohort of patients with RBBB and HF [9]. With the exception of NYHA class, patients in these trials did not derive any clear significant benefit from CRT. Authors, thereby, concluded that there is few data supporting the use of CRT in patients with RBBB. Since RBBB was proved to be an important predictor of mortality in HF patients as much as LBBB, it seems important to clarify the place of CRT in patients with RBBB and HF. Numerous small studies have evaluated the presence of viability as a predictor of response to CRT in ischemic and non ischemic failing hearts $[5,6]$. Long term response to CRT may be explained by the presence of myocardial viability in patients with LV dysfunction. Moreover, presence of viability in the region of the pacing lead has been recently showed to be a predictor of acute response to CRT [10]. This factor has never been evaluated in patients with RBBB. This preliminary report describes our experience in such patients.

\section{Methods}

From June 2006 to September 2007, we studied 4 consecutive patients with RBBB underwent CRT implantation. The study was approved by our local ethics committee. Inclusion criteria were as follow: (1) RBBB (QRS > $120 \mathrm{~ms}$ ), (2) NYHA functional class III and IV, (3) chronic LV systolic dysfunction (LV ejection fraction $\leq 35 \%$ ), (4) basal LV dyssynchrony $\geq 50 \mathrm{~ms}$, and (5) optimal medical treatment for HF including angiotensin-converting enzyme inhibitors or AT1 receptor antagonists diuretics, beta-receptor blockers and spironolactone when tolerated. Patients with recent myocardial infarction ( $<6$ months), coronary revascularization procedure ( $<6$ months) and presenting standard contraindications to DSE were excluded. All patients provided informed consent and were prospectively enrolled. One day after CRT implantation, the LV lead position was assessed from a chest X-ray. Using lateral views (anterior, lateral or posterior), we determined the LV lead location. Standard 2dimensional echocardiography studies were performed within the week before CRT implantation. Echocardiographic measurements were performed by two observers blinded to patient's status using Philips Sonos 5500 or 7500 instrument with a $2.5-\mathrm{MHz}$ transducer (Philips Medical Systems, Amsterdam, The Netherlands). LV volumes and ejection fraction were measured using the modified biplane Simpson's rule. Proximal isovelocity surface area (PISA) method was used to assess MR severity and to measure the effective regurgitant orifice (ERO) area. Intraventricular delay tissue Doppler imaging (TDI) was performed from apical view analyzing septal, inferior, lateral, posterior and anterior walls. Intraventricular electromechanical asynchrony was defined as the time between the shortest and the longest electromechanical delays among the five walls. Interventricular electromechanical asynchrony was defined as the difference between the aortic and pulmonary ejection delays. All patients underwent DSE according to a low-dose infusion protocol. Patients received 5, 10, 15, and $20 \mu \mathrm{g} / \mathrm{kg} /$ min of dobutamine in 3-minute stage, with echocardiographic images recorded at each stage $[11,12]$. Heart rate and blood pressure were monitored during each stage. Criteria for stopping the dobutamine infusion included (1) hypotension (systolic blood pressure $<90 \mathrm{mmHg}$ ), (2) angina, (3) significant arrhythmias (atrial fibrillation, bigeminy, ventricular tachycardia), (4) attainment of $85 \%$ maximal predicted heart rate. The regional wall motion was assessed by the 16segments model recommended by the American Society of Echocardiography [13]. Thus, a normal or hyperkinetic segment was graded as 1, mild hypokinetic as 2, severe hypokinetic as 3 , akinetic as 4 . Stress images during dobutamine infusion showing the maximum augmentation of wall motion were compared to baseline images. A segment was considered to have contractile reserve if the wall motion improved by one grade during DSE. Viability in the region of the $\mathrm{LV}$ pacing lead was defined as the presence of viability in 2 contiguous segments. Response to CRT was defined by evidence of LV reverse remodeling ( $\geq 15 \%$ reduction in LV end-systolic volume) 3-6 months after CRT.

\section{Results}

Table 1 lists the individual baseline characteristics of enrolled patients. The four patients (mean age $75 \pm 6$ years, 2 males) had ischemic HF, were in sinus rhythm and NYHA functional III. MR was observed in 2 patients (mean

Table 1

Population demographic and echocardiographic characteristics

\begin{tabular}{|c|c|c|c|c|}
\hline & Patient 1 & Patient 2 & Patient 3 & Patient 4 \\
\hline Age (years) & 77 & 68 & 81 & 73 \\
\hline $\operatorname{Sex}(M / F)$ & M & $\mathrm{F}$ & $\mathrm{F}$ & M \\
\hline NYHA pre CRT & III & III & III & III \\
\hline NYHA post CRT & II & II & II & III \\
\hline QRS (ms) & 172 & 150 & 164 & 150 \\
\hline LV asynchronism (ms) & 80 & 75 & 160 & 135 \\
\hline LVEF pre CRT $(\%)$ & 28 & 23 & 23 & 19 \\
\hline LVEF post CRT (\%) & 33 & 28 & 51 & 19 \\
\hline ERO pre CRT $\left(\mathrm{mm}^{2}\right)$ & - & 18 & 10 & - \\
\hline ERO post CRT $\left(\mathrm{mm}^{2}\right)$ & - & 13 & 8 & - \\
\hline Akinetic segments rest $(n)$ & 9 & 8 & 10 & 8 \\
\hline Akinetic segments dobu $(n)$ & 8 & 8 & 7 & 8 \\
\hline WMSI rest & 3.6 & 3.3 & 2.6 & 3.2 \\
\hline WMSI dobu & 3.1 & 2.7 & 2.3 & 3.1 \\
\hline Viable segments $(n)$ & 6 & 6 & 4 & 1 \\
\hline$\geq 4$ viable segments $(y / n)$ & Yes & Yes & Yes & No \\
\hline Viability pacing lead (y/n) & Yes & Yes & Yes & No \\
\hline LVSV rest $(\mathrm{ml})$ & 171 & 200 & 150 & 172 \\
\hline LVSV follow up (ml) & 119 & 170 & 80 & 174 \\
\hline LVSV reduction (\%) & 30 & 15 & 47 & - \\
\hline
\end{tabular}

NYHA: New York Heart Association; CRT: cardiac resynchronization therapy; LV: left ventricular; LVEF: left ventricular ejection fraction; ERO: effective regurgitant orifice; WMSI: wall motion score index; LVSV: left ventricular systolic volume. 
ERO $=14 \pm 6 \mathrm{~mm}^{2}$ ). Three patients were responders after CRT. In these patients, mean LV end-systolic volume decreased was $31 \pm 16 \%$ (from $174 \pm 25 \mathrm{ml}$ to $123 \pm 45 \mathrm{ml}$ ). There was no change in LV end-systolic volume in the non responder patient. With regard to LV asynchronism, no clinical significant difference was found between responders and non responder $105 \pm 48 \mathrm{~ms}$ vs. $135 \mathrm{~ms}$ respectively. Patients with pre CRT MR had a change in ERO of $25 \%$ $\left(14 \mathrm{~mm}^{2}\right.$ to $\left.10.5 \mathrm{~mm}^{2}\right)$.

All responders demonstrated viability in $\geq 4$ segments ( $5 \pm 1$ segments). Moreover, those patients had viability in $\geq 2$ segments in the region of the pacing lead which was not the case in the non responder patient. The pacing lead was in the posterior region for all patients. At peak DSE responders showed reduction of WMSI of $13 \%$ in comparison to $3 \%$ for the non responder patient.

\section{Discussion}

To the best of our knowledge, this preliminary report is the first to address viability, particularly in the region of the pacing lead, as a predictor of response to CRT in patients with RBBB. Our results show that 3 patients had a response to CRT defined by $\geq 15 \%$ reduction in LV end-systolic volume. Of interest, all responders had local viability, i.e. myocardial viability in the region of pacing lead, contrasting with the non responder patient who had neither viability in the region of the pacing lead or substantial global viability. Significant electrical conduction delay (QRS $\geq 120 \mathrm{~ms}$ ) is encountered in about $30 \%$ of patients with severe systolic dysfunction. Of these patients $\geq 90 \%$ have LBBB. Companion trial reveals that only $10 \%$ of the 1520 patients studied had RBBB [14]. In subgroup analysis of Companion trial, the addition of CRT in patients with bundle branch block other than LBBB did not resulted in significant reduction of hazard ratios of primary end-point (death or hospitalization). The few studies examining the role of CRT in RBBB patients have yielded conflicting results supporting or lacking support for CRT in this setting. Other studies, however, have found that patients with RBBB and significant intraventricular mechanical delay respond to CRT [8]. Recently, Byrne et al. reported in an animal model that the magnitude of cardiac dyssynchrony in a failing heart with a pure RBBB is considerably less than in hearts with an LBBB, despite similar prolongation of the QRS [15]. In the same line, Haghjoo reported that significant intraventricular dyssynchrony was found in $63 \%$ of patients in the LBBB group as opposed to $31 \%$ of patient in the RBBB group [16]. The mechanisms explaining CRT benefice in patient with HF is related to reduction of LV dyssynchrony and corresponding stress-strain disparities and inefficient contraction of the ventricle; it is not surprising that in presence of less LV asynchrony, patients with RBBB show less benefit from CRT than patients with LBBB.

Because regional wall thickening is influenced by the extent of myocardial fibrosis, it has been hypothesized that myocardial viability could help to predict response to CRT. Using nuclear myocardial perfusion, magnetic resonance imaging and DSE, studies have demonstrated the importance of viability in predicting response to CRT $[5,6,17]$. Furthermore, in patients with transmural scar tissue in the region of the LV pacing lead, CRT did not demonstrate clinical or LV remodeling improvement at follow-up [18]. Studies evaluating the role of viability to predict response to CRT have involved a majority of patients with LBBB. This is the first report regarding viability as a predictor of response in patients with RBBB. Some studies have suggested that ischemic dilated cardiomyopathy is less likely to respond to CRT than non ischemic dilated cardiomyopathy, whereas other studies have not. However, these studies only examined outcomes based on the presence or absence of coronary artery disease as the cause of systolic heart failure. These studies did not consider, for example, the more specific possibility that infarct location influences CRT response. Whether CRT is "wide right" in RBBB remains to be determined [7]. Insufficient clinical evidences are available to reach definitive conclusions, and this will be difficult to overcome because of the underrepresentation of RBBB in systolic HF. Although CRT "non response" is likely a diverse phenomenon, there is emerging consensus that inadequate patient selection is a key element. This preliminary report suggests that patients with RBBB do not derive the same degree of benefit from CRT unless intraventricular dyssynchrony and viability in the region of the pacing lead are present. A study designed to specifically enroll patients with RBBB, congestive HF and significant LV asynchrony is required. Moreover, in such population, evidence of myocardial viability by DSE or any other valuable method (MRI, PET scan) should also be evaluated to find out if the criterion of viability in the region of the pacing lead is as important in patients with RBBB than in patients with LBBB. Such a study may identify which patients with RBBB will derive the greatest benefit from leftsided resynchronization.

\section{Conclusion}

Prospective randomized trials concluded that patients with RBBB do not derive significant benefit from CRT. However, in these studies, significant LV asynchrony was not an inclusion criterion and viability in the region of the pacing lead was not assessed. Our preliminary report suggests that in presence of significant LV asynchrony and viability in the region of the pacing lead, patients with RBBB and HF may show similar LV reverse remodeling as patients with LBBB after CRT.

\section{Acknowledgement}

The authors of this manuscript have certified that they comply with the Principles of Ethical Publishing in the International Journal of Cardiology [19]. 


\section{References}

[1] Hunt SA, Abraham WT, Chin MH, et al. ACC/AHA 2005 guideline update for the diagnosis and management of chronic heart failure in the adult: a report of the American College of Cardiology/American Heart Association Task Force on Practice Guidelines. Circulation 2005;112: e154-235.

[2] Bleeker GB, Bax JJ, Fung JW, et al. Clinical versus echocardiographic parameters to assess response to cardiac resynchronization therapy. Am J Cardiol 2006;97:260-3.

[3] Bax JJ, Marwick TH, Molhoek SG, et al. Left ventricular dyssynchrony predicts benefit of cardiac resynchronization therapy in patients with end-stage heart failure before pacemaker implantation. Am J Cardiol 2003;92:1238-40.

[4] Ypenburg C, Schalif MJ, Bleeker GB, et al. Impact of viability and scar tissue on response to cardiac resynchronization therapy in ischaemic heart failure patients. Eur Heart J 2007;28:33-41.

[5] Da Costa A, Thévenin J, Roche F, et al. Prospective validation of stress echocardiolgraphy as an identifier of cardiac resynchronization therapy responders. Heart Rhythm 2006;3:406-13.

[6] Ypenburg C, Sieders A, Bleeker GB, et al. Myocardial contractile reserve predicts improvement in left ventricular function after cardiac resynchronization therapy. Am Heart J 2007;154:1160-5.

[7] Sweeney MO. Wide right. Heart Rhythm 2005;2:616-8.

[8] Garrigue S, Reuter S, Labeque JN, et al. Usefulness of biventricular pacing in patients with congestive heart failure and right bundle branch block. Am J Cardiol 2001;88:1436-41.

[9] Egoavil CA, Ho RT, Greenspon AJ, Pavri BB. Cardiac resynchronization therapy in patients with right bundle branch block: analysis of pooled data from the MIRACLE and Contak CD trials. Heart Rhythm $2005 ; 2: 611-5$.

[10] Senechal M, Garceau P, Champagne J, et al. Usefulness of dobutamine stress echocardiography to predict acute response to cardiac resynchronisation therapy. JACC 2008;51:S-A119.
[11] deFilippi CR, Willet DL, Irani WN, et al. Comparison of myocardial contrast echocardiography and low-dose dobutamine stress echocardiography in predicting recovery of left ventricular function after coronary revascularization in chronic ischemic heart disease. Circulation 1995;92: 2863-8.

[12] Cigarroa CG, deFilippi CR, Brickner ME, et al. Dobutamine stress echocardiography identifies hibernating myocardium and predicts recovery of left ventricular function after coronary revascularization. Circulation 1993;88:430-6.

[13] Schiller NB, Shah PM, Crawford M, et al. Recommendations for quantitation of the left ventricle by two-dimensional echocardiography. J Am Soc Echocardiogr 1989;2:358-67.

[14] Bristow MR, Saxon LA, Boehmer J, et al. Cardiac-resynchronization therapy with or without an implantable defibrillator in advanced chronic heart failure. N Engl J Med 2004;350:2140-50.

[15] Byrne MJ, Helm RH, Daya S, et al. Diminished left ventricular dyssynchrony and impact of resynchronization in failing hearts with right versus left bundle branch block. J Am Coll Cardiol 2007;50: 1484-90.

[16] Haghjoo M, Bagherzadeh A, Farahani MM, et al. Significance of QRS morphology in determining the prevalence of mechanical dyssynchrony in heart failure patients eligible for cardiac resynchronization: particular focus on patients with right bundle branch block with and without coexistent left-sided conduction defects. Europace 2008;10:566-71.

[17] Chalil S, Foley PWX, Muyhaldeen SA, et al. Late gadolinum enhancement-cardiovascular magnetic resonance as a predictor of response to cardiac resynchronization therapy in patients with ischaemic cardiomyopathy. Europace 2007;9:1031-7.

[18] Ypenburg C, Martin JS, Bleeker GB, et al. Impact of viability and scar tissue on response to cardiac resynchronization therapy in ischaemic heart failure. Eur Heart J 2007;28:33-41.

[19] Coats AJ. Ethical authorship and publishing. Int J Cardiol 2009;131: $149-50$. 